\title{
Focusing Ability of a Microsize Graphene-Based Cylindrical Reflector in the THz Range Illuminated by Electromagnetic Plane Wave
}

\author{
Taner Oğuzer \\ Dept. Electrical and Electronics Eng., Dokuz Eylul \\ University, Buca, 35160 Izmir, Turkey \\ taner.oguzer@deu.edu.tr
}

\author{
Ayhan Altintas \\ Dept. Electrical and Electronics Eng., Bilkent University, \\ 06800 Ankara, Turkey \\ altintas@ee.bilkent.edu.tr
}

\begin{abstract}
The scattering of the H-polarized plane wave by a two-dimensional (2-D) parabolic reflector made of graphene placed in the free space is simulated by using the Method of Analytical Regularization (MAR) technique. The total scattering cross-section and absorption cross-section are computed, together with the field magnitude in the geometrical focus of reflector. The surface plasmon resonances are observed. Besides, the focusing ability of the reflector is studied in dependence of the chemical potential of the graphene.
\end{abstract}

Keywords—electromagnetic scattering; reflector; focusing.

\section{INTRODUCTION}

Graphene is a non-conventional material, which is a very thin (atomic) layer with adjustable electrical and chemical properties. Due to the inductive nature of the associated surface impedance, it can support the Surface Plasmon (SP) wave. This wave can be strongly reflected back from the edges of patterned graphene so that natural SP resonances similar to Fabry-Perot type resonances can occur. This phenomenon is observed at the frequencies varying from the infrared for nanosize graphene samples to the $\mathrm{THz}$ range for the microscale ones. It has the applications in the sensor systems for nano devices and also it can be used in some optical elements.

Another feature of the graphene is that its conductivity can be controlled by applying an external electrostatic biasing field which modifies graphene's chemical potential. This can be implemented by supporting the graphene with a dielectric substrate. One can apply d-c bias using this substrate. Still in basic simulations, one can take a graphene curved strip located in the free space, and also one can assume that the biasing exists in some way. In the graphene strips, the edge effects become important if the strip width gets smaller than $100 \mathrm{~nm}$. For wider strips one can disregard these edge effects and use the electron conductivity model developed for infinite graphene layer. In the $\mathrm{THz}$ range which is actively explored in today's electromagnetic-wave technology, various graphene based sources, circuits and systems are anticipated and developed [1].

The simulations of the cylindrical reflectors made up of graphene can be performed by using the electromagnetic boundary value problem (BVP). It should also include the resistive-sheet boundary condition derived previously for a zero-thickness imperfectly conducting layer.

In our case the reflector surface is taken as a 2-D geometry and only the H-polarization case is considered. In the fullwave modeling of the reflector systems, finite-difference timedomain method is the one of the possible techniques. However it needs huge number of unknowns due to the discretization of large physical domain and also has a disadvantage in the inability to satisfy the far field radiation condition. The method of moments (MoM) procedure can also be applied to arbitrary geometries. However with conventional MoM with local basis and testing functions the overall accuracy remains at the level of 2-3 digits even for medium size reflectors (up to 10 wavelengths). If better accuracy is needed or larger reflectors are interested in, one can expect non-realistic CPU times or complete failure of the code. Another alternative is the high frequency techniques like GO, PO, PTD, which work much faster however do not produce accurate full-wave results.

Another serious alternative is the MAR (see, for instance, [2-4]). In MAR, the kernel of the singular integral equations (SIE) for the current on the reflector is separated into two parts, the more singular part (usually static) and the remainder. Then the more singular part is analytically inverted by using some special techniques like the Riemann-Hilbert Problem (RHP) method. The remainder leads to the Fredholm secondkind matrix equation that provides a convergent numerical solution. The same can be achieved by choosing the global basis functions that are the orthogonal eigenfunctions of the singular part of SIE operator and using them in a MoM-like Galerkin projection algorithm. In either case the SIE-MAR technique enables more accurate full wave analysis of the reflector problems including imperfect reflectors. In [5], assuming the parabolic contour, we studied the antenna 
characteristics of the perfectly electric conducting (PEC) reflector in 2-D. In [6], assuming the elliptical contour, the scattering and the focusing problem was studied for the uniformly and non-uniformly resistive cases.

The scattering and absorption of $\mathrm{THz}$ waves by a single flat graphene strip and finite graphene-strip gratings was reduced to SIE and its Nystrom type solution was performed in $[7,8]$. Infinite graphene-strip grating in the free space was studied by the RHP technique in [9]. In these works, reflectance, transmittance and absorbance are investigated as a function of graphene and grating parameters showing the presence of SP resonances. In a more recent work [10], the bulk refractive index sensitivities of the $\mathrm{THz}$ range $\mathrm{SP}$ resonances on a micro size graphene strip were studied. In the work [11], the scattering of $\mathrm{THz}$ waves by a graphene strip was analyzed using the SIE transformed into Fourier-transform domain.

In the present study, the focusing ability of a parabolically curved graphene reflector is modeled. To simulate graphene, the previously known resistive boundary condition is used with the proper definition of the graphene conductivity using Kubo formula [12]. The corresponding SIE is obtained from the electromagnetic BVP and solved by using the RHP-based MAR solution. This type of solution provides us accurate data in the determination of the scattering characteristics. The total scattering and absorption cross sections of the parabolic graphene strip are obtained and the SP resonances are verified. Additionally, we study on the focusing ability of such a strip as a 2-D reflector as a function of the graphene parameters.

\section{FORMULATION}

The problem geometry associated with an arbitrary profile 2-D graphene reflector illuminated by an H-polarized electromagnetic plane wave is presented in Fig. 1. The cross section of the reflector is defined as a conic-section profile and can be an elliptic, parabolic or hyperbolic arc depending on the eccentricity, $e$, so that $e<1$ for an ellipse; $e=1$ for a parabola and $e>1$ for a hyperbola, similarly to [5,6]. A closed contour denoted as $C$ is the reflector contour $M$ completed with the circular arc $S$, which must have the same curvature as the reflector at the latter's edge points. This kind of smooth contour $C$ helps obtaining the regularized solution.

The rigorous formulation of the considered BVP can be stated in terms of the Helmholtz equation, the Sommerfeld radiation condition far from the reflector, the resistive boundary condition on $M$, and an edge condition such that the field energy is limited in any finite domain around the reflector edge. Collectively, these conditions guarantee the uniqueness of the problem solution [13].

The resistive boundary condition is a well-established model of a very thin penetrable sheet like a metal thinner than skin depth or very thin dielectric layer. The same boundary condition can also be used for a graphene surface. It can be written as the following pair of equations:

$$
\left[\vec{E}_{\text {tan }}^{+}+\vec{E}_{\text {tan }}^{-}\right] / 2=Z \underbrace{\vec{n} \times\left[\vec{H}_{z}^{+}-\vec{H}_{z}^{-}\right]}_{\vec{J}_{\mathrm{t}}}, \quad \vec{E}_{\text {tan }}^{+}=\vec{E}_{\text {tan }}^{-} \quad \vec{r} \in M
$$

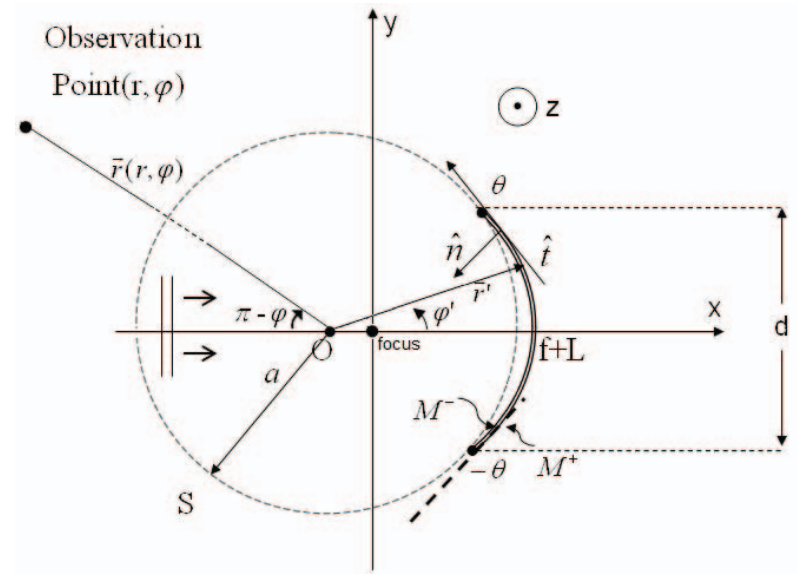

Figure 1: Problem Geometry

where the subscript " $t$ " indicates the tangential field, the superscripts ". " and "+" relate to the front and back faces of reflector, respectively, and the unit normal vector $\vec{n}$ is directed to the inside of the reflector. The unknown function $J_{t}$ is the electric surface current density.

For a graphene sheet, the resistivity is also frequently called the surface impedance, defined as $Z=1 / \sigma$. Here $\sigma$ is the graphene conductivity; it is given in [12] with the aid of Kubo formalism, as a sum of intraband and interband contributions. Although this result was derived for the infinite planar graphene layer, the given surface impedance $Z$ can also be approximately used for the finite smoothly curved surfaces.

Then by imposing the boundary conditions given in (1), the electric field integral equation (EFIE) can be derived as follows:

$$
\begin{aligned}
Z J_{\mathrm{t}}= & -\frac{i Z_{0}}{k} \frac{\partial H_{z}^{i n}}{\partial n}+\frac{i Z_{0}}{k} \frac{\partial}{\partial l} \int_{M}\left[\frac{\partial}{\partial l^{\prime}} J_{t}\left(\vec{r}^{\prime}\right)\right] G\left(\vec{r}, \vec{r}^{\prime}\right) d l^{\prime} \\
& +i k Z_{0} \int_{M} J_{t}\left(\vec{r}^{\prime}\right) \cos \left[\xi(\vec{r})-\xi\left(\vec{r}^{\prime}\right)\right] G\left(\vec{r}, \vec{r}^{\prime}\right) d l^{\prime}
\end{aligned}
$$

where the scalar Green's function $G$ is a Hankel function of the zero order and first kind satisfying the radiation condition; i.e. $G\left(\vec{r}, \vec{r}^{\prime}\right)=(i / 4) H_{0}^{(1)}\left(k_{o} R\right), R=\left|\vec{r}-\vec{r}^{\prime}\right|$. Assume that the curve $M$ can be characterized with the aid of the parametric equations $x=x(\varphi), y=y(\varphi) \quad 0 \leq|\varphi| \leq \theta$ in terms of polar angle, $\varphi$. Besides define the differential lengths in the tangential direction at any point on $M$ as $\partial l=a \beta(\varphi) \partial \varphi, \partial l^{\prime}=a \beta\left(\varphi^{\prime}\right) \partial \varphi^{\prime}$ respectively. Also $\beta(\varphi)=r(\varphi) /[a \cos \gamma(\varphi)], \xi(\varphi)$ is the angle between the normal on $M$ and the x-direction, $\gamma(\varphi)$ is the angle between the normal and the radial direction. Then we extend the surface-current density $J_{t}$ with zero value to $S$. Then the above SIE is extended to the complete contour $C$ made of $S$ and $M$ and thus is cast to a dual equation set.

To continue with the MAR-based formulation, all functions should be expended in terms of the Fourier series. Firstly the 
incident field is expressed in this manner. Furthermore we add and subtract the similar functions from the originally given ones. These are the functions at the full circle of the closed contour $C$ and introduced as follows.

$$
\begin{gathered}
A\left(\varphi, \varphi^{\prime}\right)=H_{0}^{(1)}(k R)-H_{0}^{(1)}\left(2 k a \sin \left(\left|\varphi-\varphi^{\prime}\right| / 2\right)\right) \\
B\left(\varphi, \varphi^{\prime}\right)=\cos \left(\xi(\varphi)-\xi\left(\varphi^{\prime}\right)\right) \beta(\varphi) \beta\left(\varphi^{\prime}\right) H_{0}^{(1)}\left(k\left|\vec{r}(\varphi)-\vec{r}^{\prime}\left(\varphi^{\prime}\right)\right|\right) \\
-\beta^{2}(\varphi) H_{0}^{(1)}\left(2 k a \sin \left(\left|\varphi-\varphi^{\prime}\right| / 2\right)\right)
\end{gathered}
$$

The functions $A$ and $B$ have also continuous first derivatives, and their second derivatives with respect to $\varphi$ and $\varphi$ ' have only logarithmic singularity and hence belong to $L_{2}$. Therefore their Fourier coefficients decay as $O\left(|n|^{-1.5-\varepsilon}|m|^{-1.5-\varepsilon}\right)$ on the curve $C$ and can be efficiently computed by the Fast Fourier Transform algorithm. Then the discretized version of the SIE and the zero current condition on aperture $S$ give us a dual series equation. Then semiinversion based on the MAR approach using the RHP technique finally produces an algebraic equation system $[5,6]$. This infinite matrix equation is of the Fredholm second kind hence the Fredholm theorems guarantee the existence and the uniqueness of the solution and also the convergence of the approximate numerical solution when truncating the system with progressively larger sizes.

\section{SCATTERING AND ABSORPTION CHARACTERISTICS}

The scattered magnetic and electric fields in the far zone from the reflector take the form $H_{z}^{s c}=\sqrt{2 / i \pi k r} e^{i k r} \phi(\varphi)$ and $E_{\varphi}^{s c}=\eta_{0} H_{z}^{s c}$, respectively. On the other hand, the total scattering cross section (TSCS) can be obtained by using the following equation:

$$
\sigma_{t s c}=\int_{0}^{2 \pi}\left|H_{z}^{s c}\right|^{2} r d \varphi
$$

Another characteristic is the absorption cross section (ACS). It can be obtained from the negative direction of the total outward power from the reflector at the far zone. Because no power flow can be possible in the radial direction and only power is due to the absorption from reflector conductivity [14]. Then ACS can be found as follows:

$$
\sigma_{a b s}=-\int_{0}^{2 \pi}\left|H_{z}^{s c}+H_{z}^{i n}\right|^{2} r d \varphi
$$

\section{NUMERICAL RESULTS}

The numerical accuracy and convergence of the given formulation had already been verified in [6] for the complex source beam illumination. In the current case, the problem parameters are different and we would like to analyze both the $\mathrm{H}$-polarized plane wave scattering and the focusing ability of the graphene reflector with respect to the incident wave. Here, we use TSCS and ACS as defined by (5) and (6).

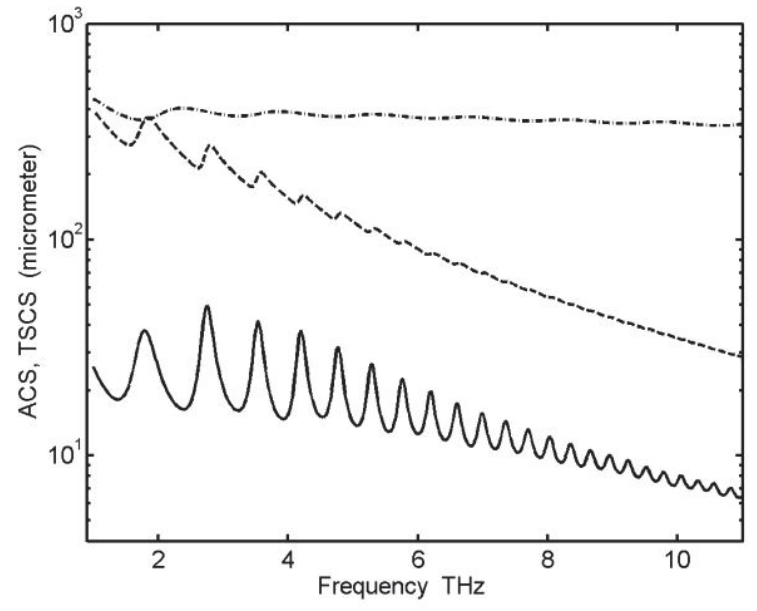

Fig. 2: Solid line: ACS and dashed line: TSCS for $\mu_{c}=1$. The dashed-dotted line: TSCS for the near-PEC case. The problem parameters are $f=5 \lambda, d=200$ $\mu \mathrm{m}, T=300 \mathrm{~K}, \tau=1 \mathrm{ps}$.

In Fig. 2, both ACS and TSCS are plotted as a function of frequency and the oscillations on the plots due to the SP resonances are observed, and the effect of the SP resonances is dominant in ACS behavior. Note that if the frequency is getting higher, then the graphene reflector becomes more and more transparent because its surface impedance $Z$ is dominated by the imaginary part, which grows up approximately linear in frequency and greatly exceeds the free-space impedance value (see [7-9,12]).

We also use another parameter presenting the amount of focusing. This is the ratio of the total field magnitude at the geometrical focus point of the parabola to the magnitude of the incident plane wave i.e. $\left|H_{z}^{\text {focus }}\right| /\left|H_{z}^{\text {in }}\right|$.

In Fig. 3, the focusing parameter is plotted for various chemical potential $\left(\mu_{c}\right)$ values. It is seen that the growth in $\mu_{c}$

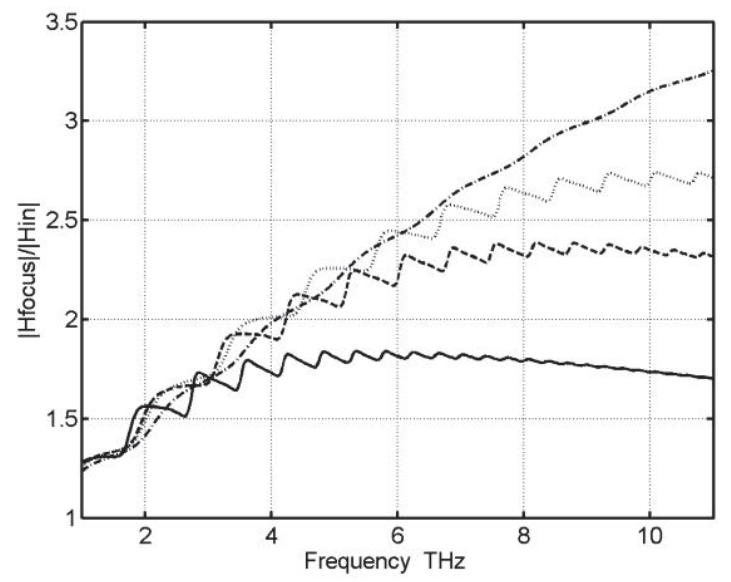

Fig. 3: The ratio of the total $\mathrm{H}$ field magnitude to the incident $\mathrm{H}$ field magnitude at focus point. Solid line: $\mu_{c}=1$, dashed Line: $\mu_{c}=2$, dotted line: $\mu_{c}$ $=3$. Dashed-dotted line: near-PEC result. The problem parameters are $f=5 \lambda, d$ $=200 \mu \mathrm{m}, T=300 \mathrm{~K}, \tau=1 \mathrm{ps}$. 
gradually increases the focusing ability especially at higher frequencies. Note that higher values of chemical potential lead to the lower values of the surface impedance of the graphene that makes it better conducting and hence the curves are closer to the PEC reflector case.

\section{CONCLUSIONS}

A 2-D graphene reflector in the free space, with arbitrary conic section profile, illuminated by an H-polarized plane wave was analyzed using the MAR approach. The results showed that a decrease in the inductive surface impedance due to the higher chemical potential of the graphene causes an enhancement in the focusing ability.

\section{REFERENCES}

[1] T. Otsuji, S. A. Boubanga Tombet, A. Satou, H. Fukidome, M. Suemitsu, E. Sano, V. Popov, M. Ryzhii, and V. Ryzhii, "Graphenebased devices in terahertz science and technology," J. Phys. D: Appl. Phys., vol. 45, no 30, pp. 303001/9, 2012.

[2] D. Colak, A. I. Nosich, and A. Altintas, "Radar cross section study of cylindrical cavity-backed apertures with outer or inner material coating: the case of E-polarization," IEEE Trans. Antennas Propagat., vol. 41, no 11, pp. 1551-1559, 1993.

[3] D. Colak, A. I. Nosich, and A. Altintas, "Radar cross-section study of cylindrical cavity-backed apertures with inner or outer material coating: the case of H-polarization," IEEE Trans. Antennas Propagat., 1995, vol. 43, no 5, pp. 440-447.

[4] A. I. Nosich, Y. Okuno, and T. Shiraishi, "Scattering and absorption of E and H-polarized plane waves by a circularly curved resistive strip," Radio Science, vol. 31, no 6, pp. 1733-1742, 1996.

[5] T. Oguzer, A. I. Nosich, and A. Altintas, "Analysis of arbitrary conic section profile cylindrical reflector antenna, H-polarization case," IEEE Trans. Antennas Propagat., vol. 52, no 11, pp. 3156-3162, 2004.
[6] T. Oğuzer, A. Altintas, and A. I. Nosich, "Analysis of the elliptic-profile cylindrical reflector with a non-uniform resistivity using the complex source and dual-series approach: H-polarization case," Opt. Quant. Electron., vol. 45, no 8, pp. 797-812, 2013.

[7] M. V. Balaban, O. V. Shapoval, and A. I. Nosich, "THz wave scattering by a graphene strip and a disk in the free space: integral equation analysis and surface plasmon resonances," IOP J. of Optics, vol. 15, no 11, pp. 114007/9, 2013.

[8] O. V. Shapoval, J. S. Gomez-Diaz, J. Perruisseau-Carrier, J. R. Mosig, and A. I. Nosich, "Integral equation analysis of plane wave scattering by coplanar graphene-strip gratings in the THz range," IEEE Trans. Terahertz Science Technol., vol. 3, no 5, pp. 666-673, 2013.

[9] T. L. Zinenko, "Scattering and absorption of terahertz waves by a freestanding infinite grating of graphene strips: analytical regularization analysis," IOP J. of Optics, vol. 17, no 5, pp. 055604/8, 2015.

[10] O. V. Shapoval and A. I. Nosich, "Bulk refractive-index sensitivities of the THz-range plasmon resonances on a micro-size graphene strip," $J$. Phys. D: Appl. Phys., vol. 49, no 5, pp. 055105/8, 2016.

[11] V. Gerasik, M. S. Wartak, A. V. Zhukov, and M. B. Belonenko, "Free electromagnetic radiation from the graphene monolayer with spatially modulated conductivity in THz range," Mod. Phys. Lett. B, vol. 30, pp.. 1650185/9, 2016.

[12] G. W. Hanson, "Dyadic Green's functions and guided surface waves for a surface conductivity model of graphene" J. Appl. Phys., vol 103, no. 6, pp. 064302/8, 2008.

[13] D. Colton and R. Kress, Integral Equation Method in Scattering Theory, Wiley Publ., 1983.

[14] M. Born and E. Wolf, Principles of Optics, $6^{\text {th }}$ edition, Pergamon Press, 1980. 